Z Herz-Thorax- Gefäßchir 2010 • 24:5-5

DOI 10.1007/s00398-009-0759-0

Online publiziert: 24. Januar 2010

(c) Springer-Verlag 2010

F. Beyersdorf

Abteilung Herz- und Gefäßchirurgie, Albert-Ludwigs-Universität Freiburg

\title{
39. Jahrestagung der DGTHG - Motor einer guten Entwicklung in die Zukunft
}

Die diesjährige 39. Jahrestagung der Deutschen Gesellschaft für Thorax-, Herz- und Gefäßchirurgie in Stuttgart wird neben dem wissenschaftlichen Programm erneut einen besonderen Schwerpunkt auf Vermittlung und praktische Demonstration neuer Operationstechniken legen. Das „training village“ soll deshalb alle Tagungsteilnehmer in direkten Kontakt mit denjenigen Techniken bringen, die in der Zukunft der Herzchirurgie einen immer größeren Raum einnehmen werden.

Parallel hierzu haben sich auch die beiden Zeitschriften der Deutschen Gesellschaft für Thorax-, Herz- und Gefäßchirurgie weiter entwickelt: auf der einen Seite das wissenschaftliche Periodikum The Thoracic and Cardiovascular Surgeon in englischer und auf der anderen Seite die Zeitschrift für Herz-, Thorax- und Gefäßchirurgie in deutscher Sprache. Beide Publikationen haben sich die weitere Fortbildung zum Ziel gesetzt.

Angesichts eines immer breiter werdenden Spektrums der Thorax-, Herzund Gefäßchirurgie ist auch bei der Organisation der Tagungen eine wichtige Entwicklung eingeleitet worden: neben die traditionelle Jahrestagung unserer Gesellschaft im Februar tritt in diesem Jahr zum zweiten Mal die Fokustagung Herz, die andere, bisher über das Jahr verteilte Aktivitäten bündelt, wie z.B. die herzchirurgische Intensivmedizin, die extrakorporale Zirkulation und mechanische Kreislaufunterstützung und die elektrophysiologischen Therapien.

Ich danke den aktiven Mitgliedern unserer Fachgesellschaft dafür, dass sie alle zusammen ebenfalls Motor dieser guten
Entwicklung unseres Fachgebiets in die Zukunft sind. Die Zeitschrift für Herz-, Thorax- und Gefäßchirurgie spielt hierbei eine bedeutende Rolle und mein Dank gilt den Herausgebern für ihren unermüdlichen Einsatz zu deren Weiterentwicklung, um so den Bedürfnissen der Mitglieder unserer Fachgesellschaft Rechnung zu tragen.

Auch die vorliegende Ausgabe enthält wieder wichtige Beiträge aus unserem gesamten Fachgebiet und ich bin sicher, dass Sie durch deren Lektüre für Ihre tägliche praktische Arbeit profitieren.

Mit den besten Wünschen bin ich Ihr<smiles>O=C1CCCC1</smiles>

Univ.- Prof. Dr. Dr. h. c. F. Beyersdorf

\section{Korrespondenzadresse}

Prof. Dr. Dr. h.c. F. Beyersdorf

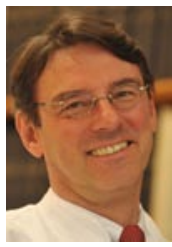

Abteilung Herz- und Gefäßchirurgie, Albert-LudwigsUniversität Freiburg Hugstetterstraße 55 , 79106 Freiburg friedhelm.beyersdorf@ uniklinik-freiburg.de 